\title{
CARACTERIZAÇÃO E AVALIAÇÃO DE FRUTOS DE ACEROLEIRA ${ }^{1}$
}

\author{
PAULA CRISTINA CARVALHO LIMA², BIANCA SARZI SOUZA', \\ PAULO SÉRGIO SOUZA ${ }^{4}$, SILMARA DA SILVA BORGES 5 , \\ MATEUS DONIZETTE OLIVEIRA DE ASSIS
}

RESUMO - Este trabalho teve por objetivo avaliar características físicas e químicas em aceroleiras provenientes de seis plantas encontradas no IFSULDEMINAS - Câmpus Muzambinho-MG, para identificar plantas com melhores qualidades genéticas. Foram selecionadas seis plantas de acerolas, e os frutos foram colhidos em quatro épocas. Em cada planta, foi feita uma amostragem com três repetições, por época, sendo cada repetição composta por 10 frutos. Foram realizadas as seguintes análises físicas: massa fresca, coloração, diâmetros longitudinal (DL) e transversal (DT), relação DL/DT, e químicas: teor de sólidos solúveis (SS), acidez titulável (AT), relação SS/AT, pH e teor de ácido ascórbico. As médias dos tratamentos, quando significativas, foram comparadas pelo teste de Tukey, a 5\% de probabilidade. Os resultados médios de massa por fruto variam de 4,03 a 7,10 g, já as médias do índice de formato do fruto variam de 0,84 a 0,92 , constatando-se que o fruto é uma drupa subglobosa. Os diâmetros transversal e longitudinal oscilam, respectivamente, de 16,67 a 23,86 mm e 15,39 a 20,06 mm, e a coloração da casca foi mais vermelha nas acerolas oriundas da planta 1 . Os teores de sólidos solúveis variam de 5,48 a $7,90^{\circ} \mathrm{Brix}$ e, na relação $\mathrm{SS} / \mathrm{AT}$, houve variação de 5,92 a 8,31. O pH variou de 3,54 a 3,76, a acidez total titulável de 0,70 a 1,14 g. $100 \mathrm{~g}^{-1} \mathrm{e}$ os teores de ácido ascórbico variaram de 1.114,07 a 1.456,22 mg.100 g-1. Observou-se que, de acordo com as características físicas, a planta 6 apresentou-se mais adequada para uma futura propagação. Em relação às variáveis químicas avaliadas, as acerolas oriundas da planta 1 apresentaram bons teores de ácido ascórbico e boa relação SS/AT, atendendo ao indicado para o suco de acerola.

Termos para indexação: Malpighia emarginata D.C., acerola, ácido ascórbico.

\section{CHARACTERIZATION AND EVALUATION OF FRUITS OF WEST INDIAN CHERRY}

\begin{abstract}
This study aimed to evaluate physical and chemical characteristics in West Indian cherry from six plants found in the IFSULDEMINAS - Campus Muzambinho, MG, to identify plants with the best genetic qualities. It was selected six plants and the fruits were collected in four dates for evaluation. In each plant, sampling was done with three replicates per date, with each replicate consisting of 10 fruits. It was realized the following physical analyzes: fresh mass, coloring, longitudinal and transverse diameter, and the ratio LD/TD, and chemical analyzes: soluble solids (SS), titratable acidity (TA), ratio SS/TA, $\mathrm{pH}$ and ascorbic acid content. The mean of treatments, when significant, were compared by Tukey test at $5 \%$ probability. The average results of fruit mass range from 4.03 to $7.10 \mathrm{~g}$ in the index means the fruit can vary from 0.84 to 0.92 noting that the fruit is a subglobose drupe. The transverse and longitudinal diameter ranging respectively from 16.67 to $23.86 \mathrm{~mm}$ and 15.39 to $20.06 \mathrm{~mm}$ and peel color was redder in fruits from Plant 1. The soluble solids ranging from 5.48 to $7.90{ }^{\circ}$ Brix and SS/TA ratio variation was from 5.92 to 8.31 . The $\mathrm{pH}$ ranged from 3.54 to 3.76 , titratable acidity from 0.70 to $1.14 \mathrm{~g} .100 \mathrm{~g}^{-1}$ and ascorbic acid

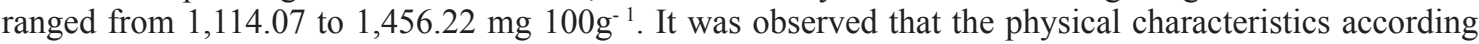
to the plant 6 showed to be more suitable for further propagation. Regarding the chemical parameters, the fruits derived from plant 1 showed good levels of ascorbic acid and good SS/TA ratio, given the indicated for the West Indian cherry juice.
\end{abstract}

Index terms: Malpighia emarginata DC, West Indian cherry, ascorbic acid.

\footnotetext{
1(Trabalho 336-13). Recebido em: 16-09-2013. Aceito para publicação em: 06-05-2014.

${ }^{2}$ Mestranda do programa de pós-graduação em Ciências Agrárias - Fisiologia Vegetal, UFV. E-mail: paulinhahlima@yahoo.com.br. ${ }^{3}$ Eng. Agr ${ }^{\mathrm{a}}$, Prof ${ }^{\mathrm{a}}$. Dr ${ }^{\mathrm{a}}$. Instituto Federal de Educação Ciência e Tecnologia do Sul de Minas Gerais - Câmpus Muzambinho. E-mail: bianca.souza@muz.ifsuldeminas.edu.br.

${ }^{4}$ Eng. Agr, Prof. Dr. Instituto Federal de Educação Ciência e Tecnologia do Sul de Minas Gerais - Câmpus Muzambinho. E-mails: paulo.souza@muz.ifsuldeminas.edu.br

${ }^{5}$ Licenciada em Ciências Biológicas. Instituto Federal de Educação Ciência e Tecnologia do Sul de Minas Gerais - Câmpus Muzambinho. E-mail: silmaraborges2008@hotmail.com

${ }^{6}$ Licenciado em Ciências Biológicas. Instituto Federal de Educação Ciência e Tecnologia do Sul de Minas Gerais - Câmpus Muzambinho.Email: mateus_muzambinho@yahoo.com.br
}

Rev. Bras. Frutic., Jaboticabal - SP, v. 36, n. 3, p. 550- 555, Setembro 2014 


\section{INTRODUÇ̃̃O}

A aceroleira é uma planta frutífera originária das Antilhas, Norte da América do Sul e Central, que vem apresentando boa adaptação em diversos países, sendo, sobretudo, cultivada no Brasil, Porto Rico, Cuba e Estados Unidos. Seu potencial como fonte natural e sua capacidade de aproveitamento industrial têm atraído o interesse de fruticultores e passou a ter importância econômica em varias regiões do Brasil (MAIA et al., 2007). Por isso, a valorização dos frutos de cada região, nas mais diversas formas de consumo, aliando seu uso na fruticultura, é uma alternativa de geração de renda para as populações locais. $\mathrm{O}$ interesse na caracterização das propriedades desses frutos tem crescido a cada dia, ocasionando aumento no número de pesquisas nessa área (ROESLER et al., 2007).

A seleção de clones é a maneira mais eficiente para suprir a demanda por variedades de uma espécie economicamente importante com um resultado que pode ser visualizado em curto prazo; e esta tem sido a principal metodologia adotada nos programas de melhoramento da acerola. Por ser a aceroleira uma espécie que pode ser propagada vegetativamente, o genótipo de cada planta pode ser transmitido integralmente através das gerações (NETO et al., 2012).

Em geral, os programas de melhoramento são realizados com base na avaliação de características agronômicas da planta e físico-químicas de qualidade dos frutos. Porém, outros parâmetros também vêm sendo avaliados devido uma mudança observada nos hábitos alimentares da população, como a busca por alimentos mais saudáveis que possam trazer benefícios à saúde. Os fitonutrientes antioxidantes, como a vitamina $\mathrm{C}$ e os compostos fenólicos, são capazes de combater os radicais livres envolvidos nos processos degenerativos celulares e, assim, fortalecer o sistema imunológico dos animais (OLSSON et al., 2006).

Atualmente, há uma grande preocupação dos consumidores sobre a importância de escolher alimentos funcionais como um meio de prevenção de doenças e na melhoria da qualidade de vida. As frutas são incluídas neste tipo de alimentos, já que elas são amplamente aceitas pelos consumidores e são fontes importantes de compostos antioxidantes, vitaminas e outros nutrientes (SANTOS et al., 2012).

Buscando uma alimentação saudável, cada vez mais os consumidores adquirem informações sobre o que consomem (SILVA et al., 2013). A fruta apresenta-se atrativa pelo seu sabor agradável e destaca-se por seu reconhecido valor nutricional, principalmente como fonte de vitamina $\mathrm{C}$, vitamina A, ferro, cálcio e vitaminas do complexo B (tiamina, riboflavina e niacina). Consumida tanto in natura como industrializada, sob a forma de sucos, sorvetes, geleias, xaropes, licores, doces em caldas, entre outras (FERREIRA et al., 2009). Outra forma de agregar valor à produção de acerola é processála para a produção de bebidas alcoólicas e não alcoólicas (SEGTOWICK, 2013).

Portanto, o presente trabalho tem como objetivo avaliar características físicas e químicas em seis acessos de aceroleiras propagadas por sementes, para identificar plantas com melhores características genéticas e para eventuais propagações assexuadas.

\section{MATERIAL E MÉTODOS}

O experimento foi conduzido no IFSULDEMINAS- Câmpus Muzambinho, Minas Gerais $\left(21^{\circ} 18^{\prime} 00^{\prime \prime} \mathrm{S}\right.$ e $46^{\circ} 30^{\prime} 00^{\prime \prime} \mathrm{W}$, a uma altitude de 1.033 metros). Foram selecionadas 6 plantas de acerolas (IFSM1 a IFSM6), e os frutos foram colhidos em quatro épocas (06-12-2011; 05-01; 06-03 e 03-04-2012). Em cada planta, foi feita uma amostragem com três repetições, por época, sendo cada repetição composta por 10 frutos. Após a colheita, em cada época, os frutos foram levados para o Laboratório de Bromatologia e Água do Instituto, para a realização das seguintes avaliações: massa fresca, coloração, diâmetros longitudinal e transversal. Após a realização das análises físicas, os frutos foram despolpados manualmente e do extrato foram retiradas amostras para a determinação do teor de sólidos solúveis, acidez titulável, $\mathrm{pH}$ e ácido ascórbico.

Os métodos utilizados para as análises físicas foram: massa fresca, determinada através de balança com capacidade para $1.200 \mathrm{~g}$ e precisão de $0,1 \mathrm{~g}$; coloração, determinada utilizando-se de colorímetro Minolta Croma Meter, que se expressa segundo o sistema proposto pela Commission Internacionale de L'Eclaraige (CIE) em L*a*b* (color space), a coloração foi relatada pelos parâmetros: luminosidade, ângulo hue ou de cor e cromaticidade (Minolta CR); diâmetro longitudinal (DL) e transversal (DT), expressos em milímetros e obtidos com o auxílio de um paquímetro (Insight); posteriormente, calculou-se a relação DL/DT para indicar o formato dos frutos.

Os métodos utilizados para as análises químicas foram: os teores de sólidos solúveis (SS) foram determinados em refratômetro digital (Atago PR 101), onde os resultados foram expressos em ${ }^{\circ}$ Brix (AOAC, 2012); a acidez titulável foi 
determinada a partir de amostras de 10 gramas de polpa homogeneizada que foram tituladas com solução padronizada de hidróxido de sódio a $0,1 \mathrm{M}$, até que estas atingissem $\mathrm{pH} 8,1$, e os resultados foram expressos em gramas de ácido cítrico por 100 gramas de polpa (AOAC, 2012); a relação sólidos solúveis/acidez titulável (ratio ou índice de maturidade) é um indicativo que se refere ao sabor do fruto, sendo calculada a partir da determinação dos parâmetros envolvidos; o pH foi determinado a partir da mesma amostra obtida para a determinação dos teores de sólidos solúveis, e para sua análise utilizou-se um potenciômetro (AOAC, 2012); o teor de ácido ascórbico foi quantificado em homogenato de 1 grama da polpa diluído com volume suficiente de ácido oxálico a 5\%,e posteriormente titulado com reagente de Tillman (2,6 diclorofenolindofenol de sódio a 0,2\%) (AOAC, 2012).

O experimento foi conduzido em delineamento inteiramente casualizado, com 12 repetições (três amostragens em quatro épocas). As médias dos tratamentos, quando significativas, foram comparadas pelo teste de Tukey, a 5\% de probabilidade, baseado na Análise de Variância (ANAVA), utilizando-se do programa estatístico SISVAR (FERREIRA, 2011).

\section{RESULTADOS E DISCUSSÃO}

De acordo com as análises físicas realizadas (Tabela 1), a massa fresca média dos frutos variou de 4,03 g nas acerolas oriundas da planta 1, a 7,10 g nas acerolas oriundas da planta 6 (IFSM6). Os valores encontrados para os frutos de aceroleiras neste estudo estão dentro do intervalo proposto por vários autores na literatura, estando próximos aos encontrados por Santos et al. (2012), onde a massa dos frutos estudados variaram de 5,45 g a 7,21 g, porém são superiores aos encontrados por Freire et al. (2006), que obtiveram valores alternando entre 2,33 e 6,27 $\mathrm{g}$, enquanto estudavam as características físicas de acerolas oriundas de pomares de diferentes regiões do Estado da Paraíba. Tais divergências, provavelmente, são devidas às diferenças no material genético, além das condições edafoclimáticas dos locais de cultivo.

Segundo Berilli et al. (2007), a fase de crescimento é uma etapa de desenvolvimento do fruto onde ocorrem as alterações quantitativas que resultam no aumento de peso e volume de água desse órgão. Tal fase é bastante influenciada por fatores do ambiente, como temperatura, radiação solar e precipitação, além de fatores genéticos intrínsecos de cada material vegetal.

Ainda na Tabela 1, verifica-se que o diâmetro longitudinal variou de $15,39 \mathrm{~mm}$ em acerolas oriundas da planta 1 (IFSM1) a 20,05 mm em acerolas oriundas da planta 6 (IFSM6), enquanto o diâmetro transversal dos frutos variou de $16,67 \mathrm{~mm}$ em frutos oriundos da planta 1 (IFSM1) a 23,86 $\mathrm{mm}$ em frutos oriundos da planta 6 (IFSM6). Estes valores estão abaixo dos encontrados por Santos et al. (2012), em que o diâmetro médio transversal dos frutos variou de 22,33 a 24,04 mm, enquanto o diâmetro longitudinal variou de 17,91 a 21,48 mm.

Estas variações estão dentro dos valores de tamanho indicados por Freire et al. (2006), que encontraram valores variando de 16,60 a 23,20 mm de diâmetro transversal, e 15,30 a 20,10 mm de diâmetro longitudinal.

Quanto ao índice de formato, que é o reflexo da relação diâmetro longitudinal e transversal, houve variação de 0,84 em frutos oriundos da planta 2 (IFSM2) e a 0,92 em frutos oriundos da planta 3 (IFSM3) (Tabela 1), valores estes que confirmam que a acerola é uma drupa subglobosa, sendo coerentes com os índices de formato encontrado por Maranhão (2010).

A coloração dos frutos apresentou variações significativas entre as plantas (Tabela 1). Observandose que os frutos variaram de uma coloração vermelha intensa para vermelha viva. De acordo com o ângulo Hue, observou-se maior valor da cor vermelha nos frutos oriundos da planta 1 (IFSM1). Constatouse, também, que em relação à cromaticidade, os frutos oriundos da planta 1 apresentaram maiores quantidades de pigmentos. E em relação à luminosidade, os maiores valores obtidos foram para os frutos oriundos das plantas 1 e 5 .

Em relação às variáveis químicas, os teores de ácido ascórbico na polpa variaram de 1.114,07 mg. $100 \mathrm{~g}^{-1}$ em frutos oriundos da planta 4, a 1.456,22 mg. $100 \mathrm{~g}^{-1}$ nos oriundos da planta 5 (IFSM5), porém não se observou diferença estatística entre as plantas (Tabela 2). Os valores são maiores que os encontrados por Ferreira et al. (2009), cerca de 963,01 mg.100 $\mathrm{g}^{-1}$ e Mezadri et al. (2008), que encontraram valores de ácido ascórbico entre 632 e 920 mg.100 g-1 em acerolas maduras do Distrito de Itajaí - SC. São semelhantes aos encontrados por Maciel et al. (2010), em que os teores de ácido ascórbico variaram de 750 a $1.678 \mathrm{mg} .100 \mathrm{~g}^{-1}$.

Os conteúdos de acidez titulável apresentam valores que variaram de $0,70 \mathrm{~g} .100 \mathrm{~g}^{-1}$, em frutos oriundos da planta 4, a 1,14 g.100 g-1 de ácido málico em frutos oriundos da planta 3 (IFSM3) (Tabela 2). Moura et al. (2007), na avaliação de 45 clones de acerola, encontraram valores que variaram de 0,53 g. $100 \mathrm{~g}^{-1}$ (Clone FP3) a 1,52 g.100 g-1 (II clone 37/3) 
e Ferreira et al. (2009) encontraram valores próximos a 1,33 g.100g-1 . Já Santos et al. (2012) encontraram valores menores aos obtidos no estudo, que variaram entre 0,87 g. $100 \mathrm{~g}^{-1}$ e $0,99 \mathrm{~g} .100 \mathrm{~g}^{-1}$ de ácido málico. Lima et al. (2012) consideram esse parâmetro importante do ponto de vista tecnológico, pois está diretamente relacionado à sua qualidade quanto ao atributo sabor, sendo mais atrativo para o consumo in natura.

Em relação aos sólidos solúveis (Tabela 2), observou-se uma variação de $5,48^{\circ}$ Brix em acerolas oriundas da planta 4 a $7,90^{\circ} \mathrm{Brix}$ nas oriundas da planta 1. De acordo com Chin et al. (2013), os frutos carnosos como acerola têm como característica comum a riqueza em açúcares e acidez relativamente alta. Os valores de sólidos solúveis totais encontrados foram maiores que os relatados por Santos et al. (2012), em que os valores variaram de 4,7 a $5,3^{\circ}$ Brix.
O índice de maturidade (IM) ou ratio, calculado como a de sólidos solúveis totais/acidez (SST/AT), está ligado ao sabor do fruto, que é maior em frutas doces (SANTOS et al., 2012). Observou-se boa relação SS/AT que variou de 5,92 em acerolas oriundas da planta 6 (IFSM6) a 8,31 em acerolas oriundas da planta 1 (IFSM1). Os valores estão maiores que os encontrados por Maciel et al. (2010), em que os Genótipos analisados apresentaram a razão SS/AT entre 3,79 (PL38) e 7,06 (PL34).

Quanto ao $\mathrm{pH}$, observa-se, na Tabela 2, que houve variação de 3,54 em acerolas oriundas da planta 3 a 3,76 em acerolas oriundas da planta 4 (IFSM4), semelhante aos obtidos por Freire et al. (2008), Maciel et al. (2010), Mamede et al. (2009), Moura et al. (2007) e Santos et al. (2012). De acordo com esses autores, o pH é um parâmetro que tem baixa variabilidade, fato confirmado por esta pesquisa.

TABELA 1- Massa, diâmetro transversal (DT), diâmetro longitudinal (DL), relação DL/DT, luminosidade (L*), ângulo de cor (Hue) e cromaticidade (Chroma) de frutos de seis acessos de aceroleiras (IFSULDEMINAS - Câmpus Muzambinho, 2011/2012).

\begin{tabular}{cccccccc}
\hline \multirow{2}{*}{ Plantas } & Massa $(\mathbf{g})$ & $\begin{array}{c}\text { DL } \\
\mathbf{( m m})\end{array}$ & $\begin{array}{c}\text { DT } \\
\mathbf{( m m})\end{array}$ & $\mathbf{D L} / \mathbf{D T}$ & \multicolumn{3}{c}{ Coloração } \\
\cline { 7 - 8 } IFSM1 & $4,03 \mathrm{~d}$ & $15,39 \mathrm{c}$ & $16,67 \mathrm{c}$ & $0,92 \mathrm{a}$ & $42,25 \mathrm{a}$ & $35,25 \mathrm{a}$ & $48,23 \mathrm{a}$ \\
IFSM2 & $5,93 \mathrm{~b}$ & $17,49 \mathrm{~b}$ & $21,05 \mathrm{~b}$ & $0,84 \mathrm{c}$ & $36,14 \mathrm{~b}$ & $29,90 \mathrm{~b}$ & $39,88 \mathrm{~d}$ \\
IFSM3 & $5,93 \mathrm{~b}$ & $18,95 \mathrm{ab}$ & $20,77 \mathrm{~b}$ & $0,91 \mathrm{ab}$ & $38,88 \mathrm{ab}$ & $30,70 \mathrm{~b}$ & $41,78 \mathrm{~cd}$ \\
IFSM4 & $4,55 \mathrm{~cd}$ & $17,56 \mathrm{~b}$ & $20,43 \mathrm{~b}$ & $0,86 \mathrm{c}$ & $38,28 \mathrm{ab}$ & $29,28 \mathrm{~b}$ & $46,85 \mathrm{ab}$ \\
IFSM5 & $5,58 \mathrm{bc}$ & $19,09 \mathrm{ab}$ & $21,79 \mathrm{~b}$ & $0,88 \mathrm{bc}$ & $42,42 \mathrm{a}$ & $30,53 \mathrm{~b}$ & $47,24 \mathrm{ab}$ \\
IFSM 6 & $7,10 \mathrm{a}$ & $20,06 \mathrm{a}$ & $23,86 \mathrm{a}$ & $0,84 \mathrm{c}$ & $35,77 \mathrm{~b}$ & $27,70 \mathrm{~b}$ & $44,05 \mathrm{bc}$ \\
\hline Teste F & $17,33^{* *}$ & $35,16 * *$ & $14,55 * *$ & $11,64 * *$ & $4,13 * *$ & $5,93 * *$ & $14,85 * *$ \\
\hline DMS (5\%) & 1,09 & 1,65 & 1,79 & 0,05 & 5,88 & 4,34 & 3,60 \\
\hline
\end{tabular}

*Médias seguidas pela mesma letra minúscula na coluna, para cada variável, não diferem significativamente entre si, pelo teste de Tukey, a $5 \%$ de probabilidade $(\mathrm{P}<0,05)$.

TABELA 2- Conteúdos de ácido ascórbico (AA), sólidos solúveis (SS), acidez titulável (AT), relação SS/ AT e pH em frutos de seis acessos de aceroleiras (IFSULDEMINAS - Câmpus Muzambinho, 2011/2012).

\begin{tabular}{|c|c|c|c|c|c|}
\hline Plantas & $\begin{array}{c}\mathrm{AA} \\
\left(\mathrm{mg.100g^{-1 } )}\right.\end{array}$ & $\begin{array}{c}\text { SS } \\
\left({ }^{\circ} \mathrm{BRIX}\right)\end{array}$ & $\begin{array}{c}\mathrm{AT} \\
\left(\mathrm{g.100g^{-1 } )}\right.\end{array}$ & SS/AT & pH \\
\hline 1 & $1420,21 \mathrm{a}$ & $7,90 \mathrm{a}$ & $1,02 \mathrm{ab}$ & $8,31 \mathrm{a}$ & $3,60 \mathrm{~b}$ \\
\hline 2 & $1267,57 \mathrm{a}$ & $7,07 \mathrm{abc}$ & $0,97 \mathrm{~b}$ & $7,41 \mathrm{ab}$ & $3,63 \mathrm{~b}$ \\
\hline 3 & $1283,60 \mathrm{a}$ & $7,28 \mathrm{ab}$ & $1,14 \mathrm{a}$ & $6,80 \mathrm{bc}$ & $3,54 \mathrm{~b}$ \\
\hline 4 & $1114,07 \mathrm{a}$ & $5,48 \mathrm{~d}$ & $0,70 \mathrm{c}$ & 7,06 abc & $3,76 \mathrm{a}$ \\
\hline 5 & $1465,22 \mathrm{a}$ & $6,45 \mathrm{bcd}$ & $0,97 \mathrm{~b}$ & $6,56 \mathrm{bc}$ & $3,63 \mathrm{~b}$ \\
\hline 6 & $1429,69 \mathrm{a}$ & $6,27 \mathrm{~cd}$ & $0,95 \mathrm{~b}$ & $5,92 \mathrm{c}$ & $3,58 \mathrm{~b}$ \\
\hline Teste F & $1,62 \mathrm{NS}$ & $12,59 * *$ & $14,87 * *$ & $6,68 * *$ & $8,47 * *$ \\
\hline DMS (5\%) & 436,03 & 1,00 & 0,15 & 1,30 & 0,11 \\
\hline
\end{tabular}

*Médias seguidas pela mesma letra minúscula na coluna, para cada variável, não diferem significativamente entre si, pelo teste de Tukey, a $5 \%$ de probabilidade $(\mathrm{P}<0,05)$. 


\section{CONCLUSÕES}

A seleção de plantas conduzida em plantios comerciais baseia-se, principalmente, nas características da planta e do fruto, como tamanho, sabor, coloração e rendimento de polpa. Desta forma, de acordo com as características físicas avaliadas, a planta 6 (IFSM6), por apresentar frutos maiores e mais vermelhos, apresenta-se mais adequada para uma futura propagação. Em relação às variáveis químicas avaliadas, as acerolas oriundas da planta 1 (IFSM1) apresentam bons teores de ácido ascórbico e boa relação $\mathrm{SS} / \mathrm{AT}$, atendendo ao indicado para o suco de acerola.

\section{REFERÊNCIAS}

AOAC - Association of Official Analytical Chemestry. Official methods of analysis. $19^{\text {th }} \mathrm{ed}$. Gaithersburg, 2012. 3000p.

BERILLI, S. S.; OLIVEIRA, J. G.; MARINHO, A. B.; LYRA, G. B.; SOUSA, E. F.; VIANA, A. P.; BERNARDO, S.; PEREIRA, M. G. Avaliação da taxa de crescimento de frutos de mamão (Carica papaya L.) em função das épocas do ano e graus-dia acumulados. Revista Brasileira de Fruticultura. Jaboticabal, v.29, n.1, p.11-14, 2007.

CHIN, J. F.; ZAMBIAZI, R. C.; RODRIGUES, R. da $\mathrm{S}$. Estabilidade da vitamina $\mathrm{C}$ em néctar de acerola sob diferentes condições de armazenamento. Revista Brasileira de Produtos Agroindustriais. Campina Grande, v.15, n.4, p.321-327, 2013.

FERREIRA, D. F. Sisvar: um sistema computacional de análises estatística. Ciência e Agrotecnologia. Lavras, v.35, n.6, p.1039-1042, 2011.

FERREIRA, R. M. A.; AROUCHA, E. M. M.; SOUZA, P. A.; QUIROZ, R. F.; FILHO, F. S. T. P. Ponto de colheita da acerola visando à produção industrial de polpa. Revista Verde. Mossoró, v.4, n.2, p. 13-16, 2009.

FREIRE, J. L. O.; LIMA, N. A.; FREIRE, A. L. O.; MARINOS, J. V. M. L.; DIAS, T. J.; SILVA, J. P. Biometric evaluations of acerola tree (Malpighia emarginata DC) and characterization of internal and external attributes of fruit. Engenharia Ambiental, Espírito Santo do Pinhal, v.5, p.41-52, 2008.
FREIRE, J. L. O.; LIMA, N. A.; SANTOS, F. G. B.; MARINOS, J. V. M. L. Características físicas de frutos de acerola cultivada de pomares de diferentes microrregiões do Estado da Paraíba. Agropecuária Técnica, Paraíba, v.27, n.2, p.105-110, 2006.

LIMA, R. M. T.; FIGUEIREDO, W. de; MAIA, G. A.; SOUSA, P. H. M. de; FIGUEIREDO, E. A. T. de; RODRIGUES, C. S. Estabilidade química, físico-química e microbiológica de polpas de acerola pasteurizadas e não pasteurizadas de cultivo orgânico. Ciência Rural, Santa Maria, v.42, n.2, p.367-373, 2012.

MACIEL, M. I. S.; MÉLO, E.; LIMA, V.; SOUZA, K. A.; SILVA, W. Caracterização físico-química de frutos de genótipos de aceroleira (Malpighia emarginata D.C.). Ciência e Tecnologia de Alimentos, Campinas, v.30, n.4, p.865-869, 2010.

MAIA, G. A.; SOUSA, P. H. M.; SANTOS, G. M.; SILVA, D. S.; FERNANDES, A. G.; PRADO, G. M. Efeito do processamento sobre os componentes do suco de acerola. Ciência e Tecnologia de Alimentos, Campinas, v.27, n.1, p.130-134, 2007.

MAMEDE, M. E. O.; MIRANDA, M. P. S.; RITZINGER, R.; GODOY, R. C. B.; VELOZO, E. S. Physicochemical and sensorial evaluation of new varieties of acerola. British Food Journal, United Kingdom, v.111, p.387-395, 2009.

MARANHÃO, C. M. C. Caracterização física, físico-química e química do fruto da aceroleira (Malpighia emarginata DC), variedade Okinawa, durante seu desenvolvimento. 73f. 2010. Dissertação (Mestrado em Ciência e Tecnologia de Alimentos na área de concentração de Origem Vegetal) - Universidade Federal da Paraíba, João Pessoa, 2010.

MEZADRI, T.; VILLAÑO, D.; FERNÁNDEZPACHÓN, M. S.; GARCIA-PARRILLA, M. C.; TRONCOSO, A. M. Antioxidant compounds and antioxidant activity in acerola (Malpighia emarginata DC) fruits and derivatives. Journal of Food Composition and Analysis, San Diego, v.21, p.282-290, 2008.

MOURA, C. F. H.; ALVES, R. E.; FIGUEIREDO, R. W.; PAIVA, J. R. Avaliações físicas e físicoquímicas de frutos de clones de aceroleira (Malpighia emarginata D. C.). Revista Ciência Agronômica, Fortaleza, v.38, n.6., p.52-57, 2007. 
NETO, J. C.; RABELO, M. C.; BERTINI, C. H. C. de M.; MARQUES, G. V.; MIRANDA, M. R. A. de. Caracterização agronômica e potencial antioxidante de frutos de clones de aceroleira. Revista Ciência Agronômica, Fortaleza, v. 43, n. 4, p. 713-721, 2012.

OLSSON, M. E.; ANDERSON, C. S.; OREDSSON, S.; BERGLUND, R. H.; GUSTAVSSON, K. E. Antioxidant levels and inhibition of cancer cell proliferation in vitro by extracts from organically and conventionally cultivated strawberries. Journal of Agricultural and Food Chemistry, Davis, v. 54, n. 04, p.1248-1255, 2006.

ROESLER, R.; MALTA, L. G.; CARRASCO, L. C.; HOLANDA, R. B.; SOUSA, C. A. S.; PASTORE, G. M. Atividade antioxidante de frutas do cerrado. Ciência e Tecnologia de Alimentos, Campinas, v. 27, n.1, p.53-60, 2007.
SANTOS, S. M. L.; VASCONCELOS, A. M.; OLIVEIRA, V. S.; CLEMENTE, E.; COSTA, J. M.C. Evaluation of physical and physicochemical characteristics of Malpighia emarginata D.C. from the state of Ceará. International Journal of Biochemistry. Research and Review, West Bengal, v.2, n.4, p.152-163, 2012.

SEGTOWICK, E. C. S.; BRUNELLI, L. T.; VENTURINI FILHO, W. G. Avaliação físicoquímica e sensorial de fermentado de acerola. Brazilian Journal Food Technology. Campinas, v. 16, n. 2, p. 147-154, 2013.

SILVA, M. L. S.; MENEZES, C. C.; PORTELA, J. V. F.; ALENCAR, P. E. B. S.; CARNEIRO, T. B. Teor de carotenoides em polpas de acerola congeladas. Revista Verde, Mossoró, v.8, n.1, p.170-173, 2013. 